\title{
Interaction of Mycobacterium tuberculosis with Host Cell Death Pathways
}

\author{
Lalitha Srinivasan, Sarah Ahlbrand, and Volker Briken \\ Department of Cell Biology and Molecular Genetics, University of Maryland, College Park, Maryland 20742 \\ Correspondence: vbriken@umd.edu
}

\begin{abstract}
Mycobacterium tuberculosis $(\mathrm{Mtb})$ has coevolved with humans for tens of thousands of years. It is thus highly adapted to its human host and has evolved multiple mechanisms to manipulate host immune responses to its advantage. One central host pathogen interaction modality is host cell death pathways. Host cell apoptosis is associated with a protective response to $M t b$ infection, whereas a necrotic response favors the pathogen. Consistently, Mtb inhibits host cell apoptosis signaling but promotes induction of programmed necrosis. The molecular mechanisms involved in Mtb-mediated host cell death manipulation, the consequences for host immunity, and the potential for therapeutic and preventive approaches will be discussed.
\end{abstract}

$T^{\text {hed }}$ he death of eukaryotic cells is an extremely diverse and complex event that has been categorized into multiple cell death types according to morphological features and unique cell signaling and effector molecules involved (Galluzzi et al. 2012b). Here we will give a brief overview of apoptosis, necroptosis, and pyroptosis, as these are the cell death modalities that are the most relevant in regard to interaction of pathogens with their host cell (Lamkanfi and Dixit 2010).

Apoptosis is morphologically characterized by cell shrinking, fragmentation of the nucleus, formation of membrane blebs, and apoptotic bodies. The apoptotic bodies contain cell cytoplasm and other cellular organelles and they are taken up by macrophages via receptor-mediated phagocytosis. In a process defined as efferocytosis, a macrophage phagocytoses a whole apoptotic cell. In both cases the cytoplasm and other cellular components never leave the dying cell, and hence apoptosis is immunologically silent. Important biochemical and cell signaling events that are often used to measure cellular apoptosis are the loss of mitochondrial outer membrane permeability (MOMP), cleavage and activation of members of the caspase protease family, fragmentation of chromosomal DNA, and externalization of phosphatidylserine at the cell membrane. The extrinsic apoptosis signaling pathway is activated by ligand binding to a death receptor (DR) such as TNF-receptor or Fas. Intracellular signaling events are complex but lead to the activation of caspase 8 , which will then activate the executioner caspase 3,6 , and 7 . The intrinsic apoptosis signaling pathway can be engaged by an increase in cytosolic reactive oxygen species (ROS), UV exposure, genotoxic stress, growth factor withdrawal, and others. These events will change the equilibrium of pro- and

Editors: Stefan H.E. Kaufmann, Eric J. Rubin, and Alimuddin Zumla

Additional Perspectives on Tuberculosis available at www.perspectivesinmedicine.org

Copyright (C) 2014 Cold Spring Harbor Laboratory Press; all rights reserved; doi: 10.1101/cshperspect.a022459

Cite this article as Cold Spring Harb Perspect Med 2014;4:a022459 
L. Srinivasan et al.

antiapoptotic Bcl-2 family members toward a majority of proapoptotic proteins being activated. Two of these are Bax and Bak, which after activation form a pore that results in MOMP and release of cytochrome $c$ into the cytosol. Here it associates with the apoptosis protease activating factor-1 (Apaf-1) to form the apoptosome complex, which will cleave and activate procaspase 9 . Just like caspase 8 , caspase 9 also cleaves and activates the executioner caspases 3, 6, and 7. The activated executioner caspase target and activate a wide array of cellular proteins, which are responsible for the morphological changes associated with apoptosis, such as caspase-activated DNases that cleave chromosomal DNA (Lamkanfi and Dixit 2010; Galluzzi et al. 2012a) (Fig. 1).

Historically, it was assumed that all cellular necrosis is accidental but it is now evident that most necrotic events are highly regulated by a multitude of pathways (Vanlangenakker et al. 2012). DRs and Toll-like receptors (TLRs) can induce the formation of an intracellular signaling complex containing Fas-associated death domain (FADD), caspase 8 , rest in peace 1 (RIPK1), and rest in peace 3 (RIPK3). This complex can either induce apoptosis via activated caspase 8 or necroptosis via activated RIPK3, depending on other regulatory proteins. RIPK3 leads, via activation of other signaling proteins, to the disruption of mitochondrial function and necrosis. RIPK1 knockout mice are not viable and so necrostatin-1, a very specific inhibitor of RIPK1, is commonly used to investigate RIPK1-mediated necroptosis induction. Another important modulator of a necrotic cell death pathway, referred to as parthanatos, is the poly(ADP-ribose) polymerase 1 (PARP1). PARP1 upon short-term activation actually helps to prevent apoptotic cell death via its DNA repair function. Nevertheless, continuous activation leads to massive ATP consumption that triggers mitochondrial malfunction and necrosis. PARP1 may also be activated by RIPK1, highlighting a connection with DR and TLR signaling (Vanlangenakker et al. 2012; Sridharan and Upton 2014).

Finally, pyroptosis can be regarded as a mixture between apoptosis and programmed ne- crosis because, like the latter, pyroptosis leads to cell membrane rupture and no membrane blebs or apoptotic bodies are produced but, similar to apoptosis, genomic DNA is degraded and caspase activation is required (Kepp et al. 2010; Aachoui et al. 2013). In contrast to apoptosis, pyroptosis leads to inflammation caused by the leakage of cytosolic content via damaged cell membranes and because of the caspase 1mediated generation of the secreted inflammatory cytokines IL-1 $\beta$ and IL-18. The caspase $1-$ mediated pyroptosis can be dependent on the adaptor ASC in the cases of NLRP3 and AIM2 activation or independent of ASC in the case of NLRC4-mediated activation. Caspase 11 transcription can be induced via TLR signaling after bacterial infection, and then it can either lead to stimulation of caspase 1 activity or directly induce pyroptosis. In contrast to caspase 1, caspase 11 is unable to cleave proIL- $1 \beta$ and proIL18 cytokines. The mechanism of caspase $11 \mathrm{ac}-$ tivation and its downstream targets for pyroptosis induction are unknown (Miao et al. 2011; Aachoui et al. 2013; Broz and Monack 2013; Ng and Monack 2013).

\section{HOST CELL APOPTOSIS AND CONSEQUENCES TO Mtb PATHOGENESIS}

The induction of host cell apoptosis was reported to have some direct bactericidal effects on intracellular bacterial viability (Molloy et al. 1994; Duan et al. 2002). Nevertheless, recent reports show that this is probably not a major factor during in vivo infections; instead, uptake of apoptotic cells by bystander macrophages and/or neutrophils, a process defined as efferocytosis, can lead to killing of Mtb (Fratazzi et al. 1997; Blomgran et al. 2012; Briken 2012; Martin et al. 2012; Yang et al. 2012). The killing of the bacteria inside the phagocytosed dead cell depends on recruitment and activation of the phagosomal NADPH oxidase complex, NOX2, to generate ROS (Yang et al. 2012). Another mechanism for the increased killing after efferocytosis is that $M t b$, contained within double membrane phagosomes, is unable to inhibit phagosome maturation, and consequently lyso- 


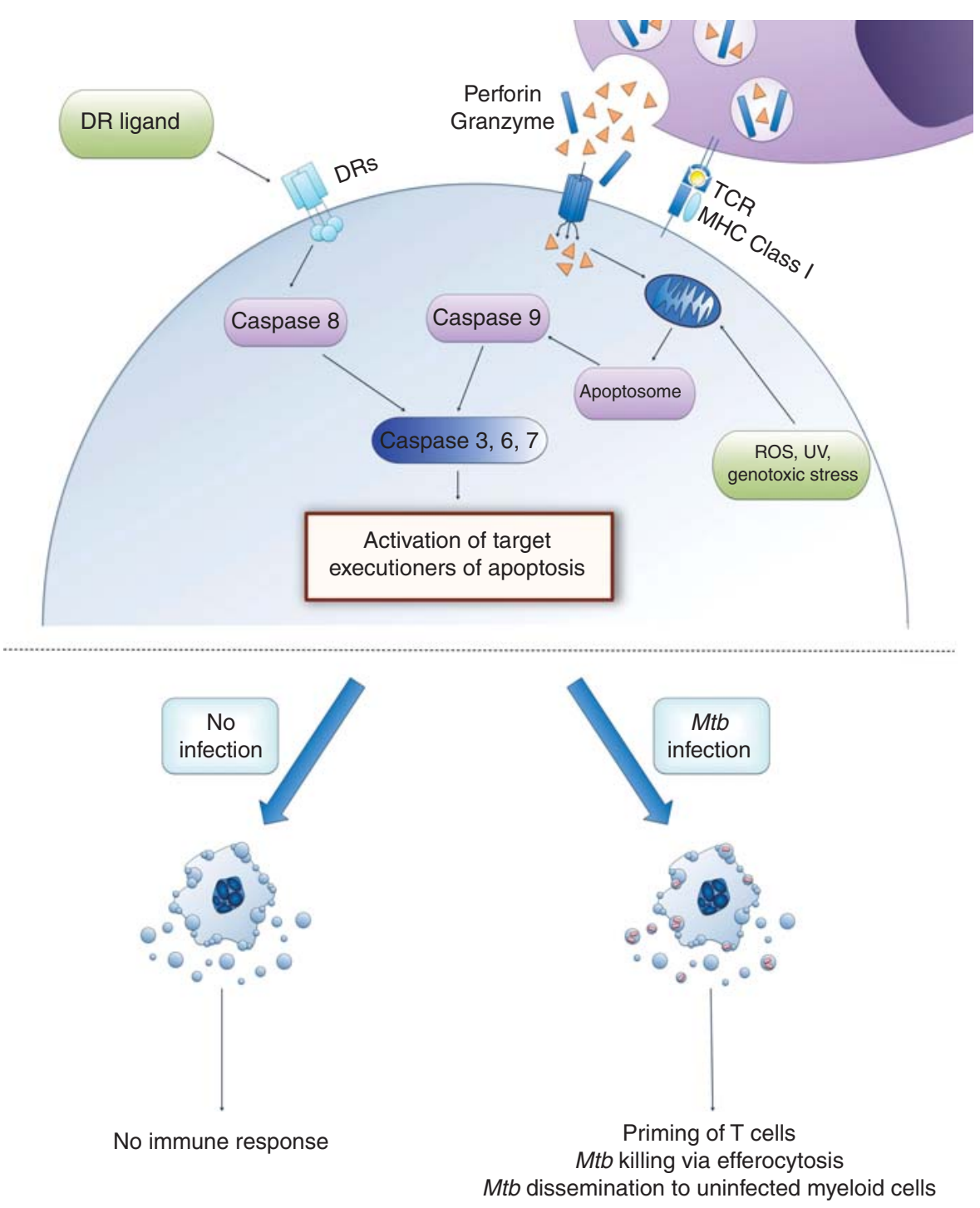

Figure 1. Host cell apoptosis pathways are classified into two main categories—extrinsic and intrinsic pathways. The extrinsic pathway of cell death is triggered by the interaction of death receptors (DRs) with specific death ligands and involves activation of caspase 8 . Intrinsic pathways are triggered by stimuli that target mitochondria and result in release of cytochrome $c$, which participates in apoptosome formation, a platform for caspase 9 activation. In addition, $\mathrm{CD} 8^{+} \mathrm{T}$ cells engage the extrinsic pathway via the Fas death receptor and the intrinsic pathway via perforin/granzyme secretion. Caspase 8 and 9 activate downstream effector caspases (caspase 3, 6, and 7), which leads to the characteristic features of apoptotic cells via activation of multiple targets. Apoptosis is immunologically silent as cellular material is enclosed within apoptotic bodies. Apoptosis of $M t b$-infected cells results in packaging of mycobacterial antigens within apoptotic bodies that, when phagocytosed by dendritic cells, allow for antigen presentation to $\mathrm{CD}^{+} \mathrm{T}$ cells via cross-presentation and the induction of a robust adaptive immune response. Additionally, uptake of dying apoptotic cells by healthy macrophages or neutrophils (efferocytosis) leads to killing of bacteria. However, $M t b$-mediated apoptosis induction was also reported to help in dissemination of bacteria during early phase of infection. 
L. Srinivasan et al.

somes and their bactericidal enzymes will fuse with the Mtb phagosome (Martin et al. 2012).

Granuloma formation is a hallmark feature of tuberculosis lung pathology (Ramakrishnan 2012; Reece and Kaufmann 2012). Compelling evidence from the zebrafish model shows that the ESX-1 secretion system, via secretion of EsxA (ESAT-6), is involved in the up-regulation of MMP9 on epithelial cells surrounding the granuloma, leading to recruitment of uninfected macrophages (Volkman et al. 2010), and that this, together with apoptosis induction of infected macrophages, helps in the dissemination of the bacteria (Davis and Ramakrishnan 2009). The beneficial effect of host cell apoptosis in the spread of bacteria and its dependence on the ESX-1 system were supported by evidence from $M t b$ in mouse lung infections showing a correlation between strains that induce increased lung cell apoptosis and bacterial growth (Aguilo et al. 2013). Consistently, the proapoptotic nuoG Mtb mutant was present in a larger number of diverse lung myeloid cells, albeit with fewer bacteria per cell, compared with wild-type $M t b$ during the early phase of aerosol mouse infection (Blomgran et al. 2012).

It is difficult to reconcile the contradictory observations of host cell apoptosis on $\mathrm{Mtb}$ pathology during the innate phase of the immune response. One explanation is that maybe during this phase both features ( protective and permissive) of host cell apoptosis come into play. This may explain why proapoptotic $M t b$ mutants do not have a virulence defect during the early phase of in vivo infections, as one would expect if only the defense features of host cell apoptosis are in effect (Hinchey et al. 2007; Velmurugan et al. 2007; Blomgran et al. 2012). Consequently, proapoptosis mutants may induce better innate immune defense responses, but they may also be more efficient in attracting uninfected macrophages, neutrophils, and dendritic cells, and hence disseminate better according to the results obtained in the zebrafish model (Davis and Ramakrishnan 2009) and by comparing dissemination of wild-type and the proapoptotic nuo $G$ mutant $M t b$ in mouse lungs (Blomgran et al. 2012).

In contrast, the long-term in vivo survival studies using proapoptosis $M t b$ mutants show that host cell apoptosis induction is beneficial to host survival overall (Edwards et al. 2001; Velmurugan et al. 2007; Sun et al. 2013). Mice infected with the nuo G Mtb mutant survive longer and have lower bacterial burden in the lungs during the persistent phase of the infection (Velmurugan et al. 2007). The knockdown of superoxide dismutase A (SodA) resulted in increased apoptosis of host cells in mouse lungs and decreased virulence of the bacteria (Edwards et al. 2001). SodA is secreted via the SecA2 secretion system in $M t b$, and a secA2 deletion mutant was attenuated in mice (Braunstein et al. 2003) and has a proapoptosis phenotype because of its defect in SodA secretion (Hinchey et al. 2007). However, the SecA2 system also affects other responses of the host cell and consequently these pleiotropic effects of the mutant make the interpretation of the cause of the in vivo attenuation difficult (Ligon et al. 2012). For example, the early growth defect of the secA 2 mutant is not caused by a lack in SodA export, because expressing a SodA protein secreted via the SecAl pathway could not reverse the phenotype (Sullivan et al. 2012). This suggests that it is not the proapoptotic feature of the SecA2 mutant that mediates that phenotype but rather the defect in the secretion of another effector (Sullivan et al. 2012). Recently, an antisense knockdown approach was successful in reducing expression of the nucleoside diphosphate kinase $(N d k)$ gene in $M t b$ and showed its importance for host cell apoptosis inhibition and virulence (Sun et al. 2013).

Another line of evidence supporting the role of host cell apoptosis in host defense is provided by studies using mouse mutants that lack genes important for either induction or repression of host cell apoptosis and their changes in susceptibility to $M t b$ infections. The Intracellular pathogen resistance 1 (Ipr1) gene is crucial for mediating host cell apoptosis instead of necrosis on $M t b$ infections ex vivo (Pan et al. 2005). The Ipr1 knockout mice are more susceptible to $M t b$ infections and induce less host cell apoptosis (Pan et al. 2005). Mice deficient in the 5lipoxygenase gene $\left(\right.$ Alox $\left.5^{-/-}\right)$cannot produce the lipoxin $\mathrm{A}_{4}\left(\mathrm{LXA}_{4}\right)$ and therefore infected macrophages undergo apoptosis instead of ne- 
crosis after $M t b$ infections (Chen et al. 2008). The Alox $5^{-/-}$mice are also more resistant to $M t b$ infection (Bafica et al. 2005; Divangahi et al. 2010). Finally, mice deficient in the protein kinase $\mathrm{R}(P k r)$ gene are more resistant to $\mathrm{Mtb}$ infections, and this correlates with higher levels of apoptosis in infected macrophages (Wu et al. 2012).

During tissue homeostasis the apoptosis of cells is immunologically silent. Nevertheless, in the context of an infection, microbial components within apoptotic bodies can, after phagocytosis, activate dendritic cells in order to induce antigen presentation and acquired immunity (Winau et al. 2004). This was first shown for a bacterial pathogen in the context of Salmonella infection (Yrlid and Wick 2000) and subsequently also for mycobacterial infection (Schaible et al. 2003). The uptake of Mtb antigens via phagocytosis of apoptotic bodies by DCs allowed for the presentation of extracellular antigens to $\mathrm{CD}^{+} \mathrm{T}$ cells, a process defined as cross-presentation (Schaible et al. 2003). Interestingly, the apoptotic bodies purified from mycobacteria-infected cells can be used to vaccinate mice and induce a protective immune response (Winau et al. 2006). This indirect pathway of generating $\mathrm{CD}^{+}{ }^{+}$-cell responses via uptake of antigen-containing apoptotic bodies by DCs was defined as the detour pathway (Winau et al. 2004). Additional evidence for the importance of host cell apoptosis induction for priming of $M t b$-specific $\mathrm{CD} 8^{+} \mathrm{T}$ cells was provided by an analysis of the immune response after infection of mice with the proapoptotic secA2 Mtb mutant (Hinchey et al. 2007). The increase in $\mathrm{CD}^{+} \mathrm{T}$-cell priming by the $\sec A 2$ mutant was abolished by overexpression of SodA, which reversed the proapoptotic phenotype of the secA2 mutant (Hinchey et al. 2007). In an elegant system, adoptive transfer of wild-type or Alox $5^{-1-}$ macrophages was used to study the impact of increased apoptosis on the induction of $\mathrm{CD}^{+} \mathrm{T}$ cells (Divangahi et al. 2010). Transfer of the Mtb-infected Alox $5^{-/-}$ macrophages (which show higher rates of apoptosis than wild-type macrophages) results in an increased and more rapid $\mathrm{CD} 8^{+} \mathrm{T}$-cell response against the mycobacterial antigen TB10.4 (Di-
Interaction of Mtb with Host Cell Death Pathways

vangahi et al. 2010). The depletion of endogenous DCs abrogates the increase in antigen presentation after adoptive transfer of the macrophages, thus showing the need for cross-presentation of $M t b$ antigens from apoptotic macrophages by DCs (Divangahi et al. 2010). Finally, aerosol infection of mice with $M t b$ and the $n u o G$ $M t b$ mutant showed an increase of lung neutrophil apoptosis after mutant infection that led to an increase in acquisition of bacteria by myeloid DCs (Blomgran et al. 2012). This resulted in a quicker and more robust priming of $M t b$ antigen 85-specific $\mathrm{CD} 4^{+} \mathrm{T}$ cells (Blomgran et al. 2012), thus highlighting the impact of host cell apoptosis induction not only on $\mathrm{CD}^{+}$but also $\mathrm{CD} 4^{+} \mathrm{T}$-cell priming. In conclusion, there is strong evidence that host cell apoptosis is an important pathway for increased host resistance to $M t b$ infection via induction of an improved acquired immune response.

\section{MANIPULATION OF APOPTOSIS PATHWAYS BY Mtb}

The literature on the inhibition of host cell apoptosis by $M t b$ has been reviewed recently (Abebe et al. 2011; Behar et al. 2011; Briken 2013). The mechanisms of inhibition include the up-regulation of host cell antiapoptosis signaling proteins such as the $\mathrm{Bcl}-2$ family member Mcl-1 (Sly et al. 2003) or the Bfl-1 protein (Kausalya et al. 2001). The Mtb-induced secretion of soluble host cell TNF-receptor 2 to inhibit TNF signaling has also been described (Balcewicz-Sablinska et al. 1998). In addition, virulent $M t b$ H37Rv strains, but not attenuated Mtb H37Ra, can inhibit TNF-mediated cell death signaling in infected cells (Keane et al. 1997). The Fas-FasL interaction is one pathway for a $\mathrm{CD} 8^{+} \mathrm{T}$ cell to induce death of target cells. $M t b$ infection leads to a reduction of Fas expression at the cell surface to inhibit T-cell-mediated killing (Oddo et al. 1998) and activates intracellular signaling pathways to suppress FasL-induced apoptosis signaling (Loeuillet et al. 2006). Finally, the neutralization of phagosomal ROS by bacterial defense mechanisms detailed below is important in suppressing TNF-mediated host cell apoptosis (Miller et al. 2010). 
L. Srinivasan et al.

\section{$M t b$ GENES INVOLVED IN HOST CELL APOPTOSIS INHIBITION}

The Mtb nuoG gene is part of a 14-gene operon (NDH-1) encoding for a type I NADH dehydrogenase whose function is important for the capacity of $M t b$ to inhibit host cell apoptosis (Velmurugan et al. 2007; Miller et al. 2010). In cells infected with the nuoG Mtb mutant, ROS accumulates in the phagosome, which leads to an increase in apoptosis (Miller et al. 2010). The detailed mechanism of how the NDH-1 system neutralizes and/or inhibits the ROS generated by the phagocyte NADPH oxidase system, NOX2, is still unknown. Catalases and superoxide dismutases are enzymes important for the detoxification of superoxides. The deletion mutant of the Mtb superoxide dismutase, SodA, is not viable but it was shown that the proapoptotic phenotype of the secretion mutant SecA2 is dependent on the secretion defect of SodA (Hinchey et al. 2007). It is thus likely that the mutants deficient in secretion of SodA lead to an increase in phagosomal ROS similar to the $n u o G$ mutant, which results in increased apoptosis. Another $M t b$ protein that targets host cell ROS is the enhanced intracellular survival (eis) protein. Nevertheless, it can inhibit not only NOX2-generated phagosomal ROS but also mitochondrial ROS via inhibition of the intracellular kinase JNK (Kim et al. 2012). eis is a secreted protein that reaches the host cell cytosol and, via its acetyltransferase activity, acetylates the phosphatase DUSP16/MKP-7 that increases its activity to dephosphorylate and hence inactivate JNK (Kim et al. 2012). The eis $M t b$ mutant induces caspase-independent apoptosis and autophagy that is different from the nuoG mutant that induces caspase-dependent apoptosis and no autophagy (Miller et al. 2010; L Srinivasan, unpubl.). Besides ROS, reactive nitrogen intermediates (RNIs) are generated in the phagosome to kill bacteria. The serine/threonine protein kinase $\mathrm{E}(\mathrm{PknE})$ of $M t b$ is up-regulated by an increase in RNIs, and without PknE there is an increase in the expression of proapoptotic members of the Bcl-2 family (Bax and Bid), a down-regulation of the antiapoptotic members such as Mcl-1, and a dephosphorylation of the prosurvival kinase Akt (Jayakumar et al. 2008; Kumar and Narayanan 2012).

A "loss-of-function" genetic screen for proapoptotic transposon mutants of $M t b$ identified the importance of two operons for host cell death inhibition (Danelishvili et al. 2010). The first operon $(R v 3654 c-R v 3660 c)$ encompasses four genes that have homology with type IV pili. Interestingly, Rv3654c is secreted into the host cell cytosol, where it binds to and cleaves the protein-associated splice factor (PSF), which is important for splicing of caspase 8 pre-mRNA (Danelishvili et al. 2010). The consequence of this interaction is that there are lower levels of caspase 8 in cells infected with wild-type Mtb compared with a $R v 3654 c$ deletion mutant (Danelishvili et al. 2010). The other antiapoptotic operon is Rv3361c-Rv3365c, which is implicated in the inhibition of cell death dependent on the inflammatory caspase 1 (Danelishvili et al. 2011). After Mtb infection, Rv3364c enters the host cell cytosol and binds to and inactivates the host cell protease cathepsin G (Danelishvili et al. 2011). How cathepsin G regulates caspase 1 activity is not known but it was hypothesized that the effect might be mediated via cathepsin $G$ cleavage and activation of the protease activated receptor- 4 , which then can induce caspase 1 activation via a NOD (nucleotide-binding oligomerization domain)-dependent pathway (Danelishvili et al. 2011) (for a detailed review, see Briken 2013).

\section{HOST CELL PROGRAMMED NECROSIS AND CONSEQUENCES TO $M t b$ PATHOGENESIS}

Host cell programmed necrosis is not detrimental to viability of intracellular $M t b$ (Molloy et al. 1994; Duan et al. 2002; Gan et al. 2008). Instead $M t b$, after infection and multiplication, induces programmed necrosis to escape the cell (Chen et al. 2006; Lee et al. 2006; Behar et al. 2010).

The host cell production of the eicosanoids, $\mathrm{PGE}_{2}$ versus $\mathrm{LXA}_{4}$, is of critical importance for the induction of apoptosis or necrosis, respectively (Behar et al. 2010). Mice deficient in 5- 
lipoxygenase cannot make $\mathrm{LXA}_{4}$ and thus are more resistant to $M t b$ infections (Bafica et al. 2005). On the other hand, mice deficient in prostaglandin E synthase (PTGES) cannot produce $\mathrm{PGE}_{2}$, and these animals are more susceptible to $M t b$ infections (Divangahi et al. 2009). Mutations in zebrafish and humans in the leukotriene A4 hydrolase (LTA4H) are associated with hypersusceptibility state to mycobacterial infections (Tobin et al. 2010). This is due to the increase in $\mathrm{LXA}_{4}$ in the absence of LTA4H, leading to increased necrosis and decreased TNF production, which favors mycobacterial replication and leads to host cell programmed necrosis and increased bacterial virulence (Tobin et al. 2010). Interestingly, the overexpression of LTA4 $\mathrm{H}$ and subsequent high levels of the leukotriene B4 (LTB4) and low levels of $\mathrm{LXA}_{4}$ also leads to an increase in host cell programmed necrosis and increased susceptibility to mycobacterial infections (Tobin et al. 2012). This is due to the induction of excessive TNF production by LTB4, which keeps bacterial replication low, but favors induction of host cell necrosis and excessive inflammation leading to tissue damage and pathology (Tobin et al. 2012; Roca and Ramakrishnan 2013). Hence at both extreme ends of the spectrum, high $\mathrm{LXA}_{4} /$ no LTB4 and high LTB4/no LXA host are very similar with high levels of host cell necrosis, host tissue pathology, and host susceptibility (Tobin et al. 2012; Roca and Ramakrishnan 2013). This is despite the fact that the bacterial numbers are quite different in the two scenarios. High $\mathrm{LXA}_{4}$ levels result in high bacterial numbers, and high LTB4 levels result in a low bacterial burden (Tobin et al. 2012; Roca and Ramakrishnan 2013). In conclusion, the Goldilocks principle applies to levels of $\mathrm{LXA}_{4}$ and LTB4 in order to produce a successful host immune response (Tobin and Ramakrishnan 2013).

\section{MANIPULATION OF PROGRAMMED NECROSIS PATHWAYS BY Mtb}

A correlation between virulence of $M t b$ and its capacity to induce mitochondrial-dependent necrosis was first proposed by studies comparing the capacities of $M t b \mathrm{H} 37 \mathrm{Rv}$ and the
Interaction of Mtb with Host Cell Death Pathways

attenuated $M t b \mathrm{H} 37 \mathrm{Ra}$ strains to disrupt mitochondrial function (Chen et al. 2006; Behar et al. 2011). Indeed only Mtb H37Rv led to an early mitochondrial transmembrane potential loss caused by mitochondrial permeability transition (MPT) pore complex. Inhibitors of MPT and mitochondrial $\mathrm{Ca}^{2+}$ influx both abrogated $M t b \mathrm{H} 37 \mathrm{Rv}$-mediated programmed necrosis induction (Duan et al. 2002; Chen et al. 2006). Other reports showed caspase-independent, programmed necrotic cell death induction after infection of cells with high bacterial numbers that did not lead to bactericidal activity (Lee et al. 2006). The threshold for host cells to undergo necrosis was determined to be between 20 and 40 bacteria per phagocyte as determined in ex vivo and in vivo studies (Lee et al. 2006; Repasy et al. 2013). This death pathway was independent of $\mathrm{Ca}^{2+}$ flux and MPT and did not require lysosomal cathepsin protease activity (Lee et al. 2006, 2011; Welin et al. 2011; Wong and Jacobs 2011). One report showed that this high bacterial burden, programmed necrosis pathway is independent of ESX-1 secretion system but requires the presence of the PhoPR two-component system for the activation of lysosomal lipases that mediate cell necrosis (Lee et al. 2011). This is in contrast to other studies, which showed that cell death induction of $M t b$-infected cells was dependent on bacterial expression of EsxA, one protein of the ESX-1 system that is involved in function of the system but is also a secreted effector itself (Welin et al. 2011; Wong and Jacobs 2011; Simeone et al. 2012). The function of the ESX-1 system was linked to escape of $M t b$ out of the phagosome at later stages of the infection, which coincided with host cell necrosis induction (Simeone et al. 2012). The Mtb EsxA protein is important in forming pores in the phagosomal membrane (Wong and Jacobs 2011), which is essential for subsequent NLRP3-inflammasome activation, because esxA Mtb mutants are deficient in activation of this inflammasome (Koo et al. 2008; Kurenuma et al. 2009; Wong and Jacobs 2011; Abdalla et al. 2012; Dorhoi et al. 2012). The activation of NLRP3 inflammasome in $M t b$-infected cells is dependent on the tyrosine kinase Syk, and this activation is 
L. Srinivasan et al.

essential for cell lysis, hence showing a necroptotic host cell death pathway (Wong and Jacobs 2011) (Fig. 2). This is consistent with a previous report showing the activation of the NLRP3 inflammasome by Syk during a fungal infection (Gross et al. 2009). Another intriguing aspect of host cell programmed necrosis during mycobacterial infections was recently revealed by studies in the zebrafish model, which showed that too low as well as too high levels of host TNF production resulted in increased necrosis and host susceptibility to infection (Roca and Ramakrishnan 2013). At too low levels of TNF, the high intracellular bacterial burden led to necrosis, whereas at too high levels of TNF the burden of bacterial per cell was actually reduced but cells were sensitized by TNF to undergo RIPK1/3-dependent necroptosis (Roca and Ramakrishnan 2013).

Virulent $M t b$ induces the production of $\mathrm{LXA}_{4}$ that inhibits cyclooxygenase 2 (COX2) expression, which is an essential enzyme in the $\mathrm{PGE}_{2}$ synthesis pathway, and thus increased $\mathrm{LXA}_{4}$ levels result in decreased $\mathrm{PGE}_{2}$ (Chen et al. 2008). $\mathrm{PGE}_{2}$ is a central inhibitor of host cell programmed necrosis via two potentially related mechanisms. First, high levels of $\mathrm{PGE}_{2}$ limit the mitochondrial membrane damage, which leads to less ROS leakage into the cytosol and hence less programmed necrosis induction (Chen et al. 2008). This effect is mediated via $\mathrm{PGE}_{2}$ binding to the E-prostanoid (EP) 2 receptor leading to protein kinase $\mathrm{A}$ activation and increased cAMP production (Chen et al. 2008). Second, $\mathrm{PGE}_{2}$ induces the recruitment of Lamp-1-positive vesicles of lysosomal origin to the cell surface to repair damaged cell membranes (Divangahi et al. 2009). On the basis of susceptibility to $\mathrm{PI} 3(\mathrm{~K})$ inhibitors it was proposed that the mechanism involves binding of $\mathrm{PGE}_{2}$ to the $\mathrm{EP} 4$ receptor leading to $\mathrm{PI}(3) \mathrm{K}$ activation. In the same study it was clearly shown that $\mathrm{PGE}_{2}$ leads to increased expression of the calcium sensor synaptotagmin 7 (Syt-7) and reduced necrosis and, vice versa, that reduction of Syt-7 via siRNA approaches inhibited plasma membrane repair (Divangahi et al. 2009). These data show that regulation of Syt7 by $\mathrm{PGE}_{2}$ is central to suppression of host cell necrosis during infection with avirulent $M t b$ strains.

Another pathway through which $\mathrm{LXA}_{4}$ facilitates programmed necrosis is via inhibition of the formation of the apoptotic cell envelope (Gan et al. 2008). The primary target of $\mathrm{LXA}_{4}$ in these events is the serine protease inhibitor, plasminogen activator inhibitor type 2 (PAI2), which is down-regulated by increasing LXA $_{4}$ levels (Gan et al. 2008). During the apoptotic envelope formation, phosphatidylserine (PS) and annexin-1 levels at the cell membrane are augmented. Annexin-1 binds to PS and is the target for the tissue transglutaminase (tTG), which cross-links annexin-1 to form a matrix that stabilizes the apoptotic envelope. Nevertheless, annexin-1 can be cleaved by an unknown cellular serine protease, which will result in a truncated annexin-1 that binds to PS but cannot be cross-linked and hence fails to support the formation of a stable apoptotic envelope (Gan et al. 2008). The result will be the induction of cellular lysis. PAI2 is a serine protease inhibitor that can inhibit the protease cleaving annexin-1. Thus, Mtb, by inhibiting PAI2 expression, facilitates programed necrosis induction (Gan et al. 2008).

As mentioned above, $M t b$ inhibits the lysosomal cell membrane repair pathway via downregulation of the calcium sensor Syt-7. There is a second cell membrane repair pathway that is not controlled by $\mathrm{PGE}_{2}$ and is dependent on Golgi-derived vesicles (Divangahi et al. 2009). Interestingly, only this pathway shuttles PS and annexin-1 to the cell membrane in order to inhibit cell necrosis and support induction of apoptosis (Divangahi et al. 2009). Hence, by targeting the PAI2 protease inhibitor, $M t b$ has evolved to find a way to functionally inhibit also this second cell membrane repair mechanism. It is fascinating that this is achieved via targeting of one central cellular signaling molecule the eicosanoid $\mathrm{LXA}_{4}$ (for review, see Behar et al. 2011). A precise programmed necrosis pathway using the available hallmark inhibitors or knockout mice (Fig. 2) has not been defined by these studies involving $\mathrm{LXA}_{4}$ and consequently it was referred to here just as programmed necrosis. 


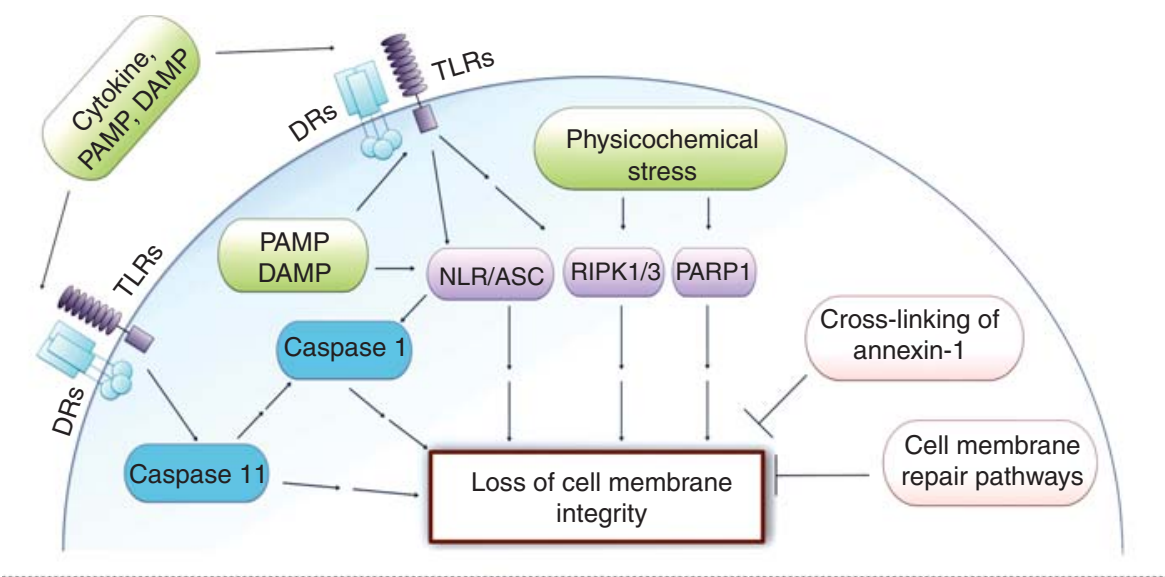

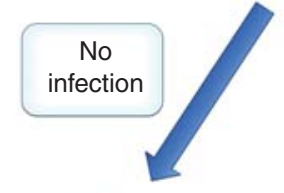
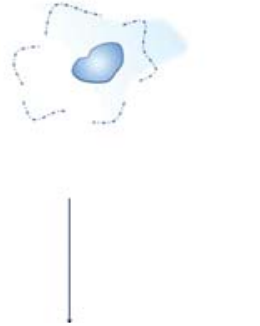

Inflammation
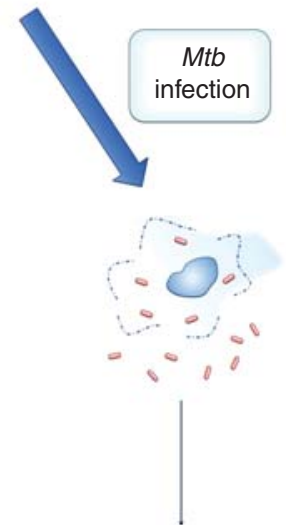

Inflammation Replication and dissemination of $M$ tb

Figure 2. Necroptosis and pyroptosis both lead to the loss of cell membrane integrity. Necroptosis can be initiated by extracellular ligands interacting with their cognate cell surface receptors (DRs, death receptors; TLR, Toll-like receptors) or by varied intracellular stress signals ( physicochemical stress). One pathway involves activation of RIPK1 and RIPK3, which leads to mitochondrial malfunction. Intracellular stress can also activate PARP1, which can signal for mitochondrial dysfunction potentially involving RIPK1 (sometimes referred to as the parthanatos death pathway). The inflammasome (NLR/ASC) may signal directly for necroptosis induction or indirectly via caspase 1 leading to pyroptosis, which also results in cell lysis but induces DNA degradation similar to apoptotic cell death. Caspase 11 can signal directly for a pyroptosis-like cell death or indirectly via NLR/ASC to activate caspase 1 -dependent pyroptosis signaling. The cell may attempt to inhibit cell membrane lysis either by recruitment of lysosomal membranes to the cell surface to repair the cell membrane or by crosslinking annexin-1 on the cell surface and thus leading to formation of a stable apoptotic envelope. Necrotic cell death is inflammatory as it leads to spilling of the intracellular contents into the extracellular milieu. Mtb has been associated with both the induction of necrosis pathways and the inhibition of the inhibitory mechanisms. Programmed necrosis of $M t b$-infected host cells leads to bacterial escape, whereas the associated inflammatory response recruits new host cells for extracellular $M t b$ to infect and replicate in, thus leading to dissemination of infection. 
L. Srinivasan et al.

\section{$M t b$ GENES INVOLVED IN HOST CELL PROGRAMMED NECROSIS INDUCTION}

There are several $M t b$ genes that are involved in the induction of host cell death (Briken 2013). Nevertheless, it is still not determined which of these or other, yet to be discovered, genes are the mediators of the $M t b$ necrotic cell death induction during the final stage of the bacterial intracellular replication cycle. An interesting candidate for a major role is the heparin-binding hemaglutinin (HBHA) protein, which is secreted into the host cell cytosol and targeted to the mitochondria to induce activation of the Bax protein (Sohn et al. 2011). This leads to the creation of a mitochondrial membrane channel resulting in the loss of membrane potential (Sohn et al. 2011). It is noteworthy that inhibition of mitochondrial membrane damage is one of the key features of PGE2 to inhibit host cell necrosis (Chen et al. 2008). Nevertheless, the Mtb HBHA protein does not seem to induce necrotic cell death but instead leads to caspase 9-dependent apoptotic cell death (Sohn et al. 2011).

The ancestral mycobacterial type VII secretion system ESX-4 has been duplicated four times during the evolution of mycobacterial species to yield ESX-1 to ESX-5 (Gey van Pittius et al. 2006; Houben et al. 2013). The EsxA protein of the ESX-1 system can form homo- and heterodimers that can insert into the host cell membrane to induce necrosis (Hsu et al. 2003; de Jonge et al. 2007; Smith et al. 2008; Welin et al. 2011). In contrast, other reports show that ectopic expression of EsxA and/or the incubation of cells with purified EsxA induce host cell apoptosis (Derrick and Morris 2007; Choi et al. 2010). There is a clear consensus though in the literature that $M t b$ or $\mathrm{Mm}$ mutants deficient in es $x A$ induce less cell death and that these mutants are also severely attenuated in the mouse and zebrafish model of tuberculosis (Abdallah et al. 2007; DiGiuseppe Champion and Cox 2007; Ramakrishnan 2012). The interpretation of results with the es $x A$ mutants in regard to the bacterial effector mediating the phenotype is difficult because deletion of es $x A$ not only affects secretion of EsxA but also of all the other ESX-1 secretion system substrates.
A functional ESX-1 system is also essential for full activation of the NLRP3-inflammasome after mycobacterial infection (Koo et al. 2008; Mayer-Barber et al. 2010; McElvania Tekippe et al. 2010; Mishra et al. 2010; Walter et al. 2010; Abdalla et al. 2012; Dorhoi et al. 2012). One report shows that this activation of NLRP3 leads to the induction of necroptosis (Wong and Jacobs 2011). The Mtb Rv3364c protein is an inhibitor of host cell cathepsin $G$ and this inhibition led to less caspase 1-dependent pyroptosis (Danelishvili et al. 2011). The ESX-5 system has also been implicated in the induction of host cell necrosis and inflammasome activation (Abdallah et al. 2011). According to the proposed model, the ESX-1 system helps Mtb to escape the phagosome following which ESX5 -secreted effector proteins participate in the induction of programmed necrosis to allow bacteria to leave the infected cell (Abdallah et al. 2011). The ESX-5 system is crucial for the secretion of the Mtb PE/PPE family of proteins (Abdallah et al. 2006, 2009). Interestingly, the PE_PGRS33 protein is transported to host cell mitochondria and induces programmed necrosis when expressed ectopically in mammalian cells (Cadieux et al. 2011). It remains to be determined what the function of this protein is in the context of live $M t b$ infection.

\section{POTENTIAL FOR THERAPEUTIC AND PREVENTIVE APPROACHES}

The fact that increased host cell apoptosis resulted in improved $\mathrm{CD}^{+}$and $\mathrm{CD} 4^{+}$T-cell priming as discussed above obviously led to efforts to improve the current vaccine, BCG (Bacillus Calmette-Guérin), by increasing its apoptosis-inducing potential. The first evidence that this strategy might be successful was provided by a BCG vaccine modified to express the Listeria monocytogenes toxin Listeriolysin $\mathrm{O}$ (LLO). The toxin is not secreted outside the infected cell but inserts into the phagosomal membrane to generate pores that allow better access of BCG proteins to the host cell cytosol, which should result in improved $\mathrm{CD} 8^{+} \mathrm{T}$-cell priming. This BCG-LLO strain also induces increased host cell apoptosis compared with wild- 
type BCG (Grode et al. 2005). Indeed, apoptotic vesicles derived from macrophages infected with this vaccine strain led to increased $\mathrm{CD}^{+}$ and $\mathrm{CD}^{+}{ }^{+}$T-cell priming (Farinacci et al. 2012). Overall the BCG-LLO strain offered better protection to mice from virulent $M t b$ challenge (Grode et al. 2005), and this vaccine is now in phase IIa human clinical trials (Kaufmann 2013). The secA2 deletion mutant in the background of an auxotroph $M t b$ vaccine strain also increases protection of mice to virulent $M t b$ challenge when compared with the original auxotroph $M t b$ vaccine strain or to BCG-vaccinated animals (Hinchey et al. 2007, 2011).

Immunotherapeutic approaches to treatment of tuberculosis are one additional approach besides traditional chemotherapy and vaccine prevention. The hypersusceptibility of zebrafish with a specific LTA4H genotype resulting in high TNF levels could be reversed by using inhibitors of the TNF-induced programmed necrosis pathway (Tobin et al. 2010; Roca and Ramakrishnan 2013). Indeed, alisporvir is an inhibitor of cyclophilin D that is released by the mitochondria via the MPT pore and is currently in phase III human trial to treat hepatitis C (Quarato et al. 2012). The other drug used in the study is desipramine, which is an antidepressant that has been given to humans for half a century. Desipramine inhibits the production of ceramides, which, at sufficiently high levels, may induce host cell necroptosis (Roca and Ramakrishnan 2013). The combination of both drugs results in synergy in suppressing virulence of mycobacterial infection in zebrafish (Roca and Ramakrishnan 2013). Human patients with a variant of the LTA4H promoter that resulted in high $\mathrm{LXA}_{4}$ levels could be successfully treated with dexamethasone, a TNFsuppressive drug (Tobin et al. 2012). Hence, inhibition of host cell necroptosis holds a lot of promise as one part of a multipronged approach to tuberculosis treatment (Tobin and Ramakrishnan 2013).

\section{CONCLUDING REMARKS}

The interaction of $M t b$ with the host in regard to cell death is complex. Currently, the research is
Interaction of Mtb with Host Cell Death Pathways

at a stage that does not allow designing a model that explains all of the data available. For example, more time and effort is needed to elucidate the ambiguous role of host cell apoptosis in $M t b$ pathogenesis. Also, how does $M t b$ regulate the induction of host cell necrosis in order to escape the cell and what are the bacterial effectors involved? Identifying these effectors may provide excellent novel drug targets because inhibiting the necrotic property of $M t b$ during the later stages of active pulmonary disease should be able to provide clear therapeutic benefits. It is now well established that $M t b$ infection of host cells involves interaction with the three major cell death pathways: apoptosis, pyroptosis, and necroptosis. It is quite likely that we will be able to gain many insights into these cell death pathways by studying a pathogen that has coevolved with humans for more than 50,000 years (Hershberg et al. 2008).

\section{ACKNOWLEDGMENTS}

Research in the Briken laboratory was supported by National Institutes of Health grant AI072584.

\section{REFERENCES}

Aachoui Y, Sagulenko V, Miao EA, Stacey KJ. 2013. Inflammasome-mediated pyroptotic and apoptotic cell death, and defense against infection. Curr Opin Microbiol 16: 319-326.

Abdallah AM, Verboom T, Hannes F, Safi M, Strong M, Eisenberg D, Musters RJ, Vandenbroucke-Grauls CM, Appelmelk BJ, Luirink J, et al. 2006. A specific secretion system mediates PPE41 transport in pathogenic mycobacteria. Mol Microbiol 62: 667-679.

Abdallah AM, Gey van Pittius NC, Champion PA, Cox J, Luirink J, Vandenbroucke-Grauls CM, Appelmelk BJ, Bitter W. 2007. Type VII secretion-mycobacteria show the way. Nat Rev Microbiol 5: 883-891.

Abdallah AM, Verboom T, Weerdenburg EM, Gey van Pittius NC, Mahasha PW, Jimenez C, Parra M, Cadieux N, Brennan MJ, Appelmelk BJ, et al. 2009. PPE and PE_PGRS proteins of Mycobacterium marinum are transported via the type VII secretion system ESX-5. Mol Microbiol 73: 329-340.

Abdallah AM, Bestebroer J, Savage ND, de Punder K, van Zon M, Wilson L, Korbee CJ, van der Sar AM, Ottenhoff TH, van der Wel NN, et al. 2011. Mycobacterial secretion systems ESX-1 and ESX-5 play distinct roles in host cell death and inflammasome activation. J Immunol 187: $4744-4753$. 


\section{Srinivasan et al.}

Abdalla H, Srinivasan L, Shah S, Mayer-Barber KD, Sher A, Sutterwala FS, Briken V. 2012. Mycobacterium tuberculosis infection of dendritic cells leads to partially caspase-1/ 11 -independent IL-1 $\beta$ and IL-18 secretion but not to pyroptosis. PLoS ONE 7: e40722.

Abebe M, Kim L, Rook G, Aseffa A, Wassie L, Zewdie M, Zumla A, Engers H, Andersen P, Doherty TM. 2011. Modulation of cell death by $M$. tuberculosis as a strategy for pathogen survival. Clin Dev Immunol 2011: 678570.

Aguilo JI, Alonso H, Uranga S, Marinova D, Arbues A, de Martino A, Anel A, Monzon M, Badiola J, Pardo J, et al. 2013. ESX-1-induced apoptosis is involved in cell-to-cell spread of Mycobacterium tuberculosis. Cell Microbiol 15: 1994-2005.

Bafica A, Scanga CA, Serhan C, Machado F, White S, Sher A, Aliberti J. 2005. Host control of Mycobacterium tuberculosis is regulated by 5-lipoxygenase-dependent lipoxin production. J Clin Invest 115: 1601-1606.

Balcewicz-Sablinska MK, Keane J, Kornfeld H, Remold HG. 1998. Pathogenic Mycobacterium tuberculosis evades apoptosis of host macrophages by release of TNF-R2, resulting in inactivation of TNF- $\alpha$. J Immunol 161: 2636-2641.

Behar SM, Divangahi M, Remold HG. 2010. Evasion of innate immunity by Mycobacterium tuberculosis: Is death an exit strategy? Nat Rev Microbiol 8: 668-674.

Behar SM, Martin CJ, Booty MG, Nishimura T, Zhao X, Gan HX, Divangahi M, Remold HG. 2011. Apoptosis is an innate defense function of macrophages against $\mathrm{Myco}$ bacterium tuberculosis. Mucosal Immunol 4: 279-287.

Blomgran R, Desvignes L, Briken V, Ernst JD. 2012. Mycobacterium tuberculosis inhibits neutrophil apoptosis, leading to delayed activation of naive CD4 T cells. Cell Host Microbe 11: 81-90.

Braunstein M, Espinosa BJ, Chan J, Belisle JT, Jacobs WR Jr. 2003. SecA2 functions in the secretion of superoxide dismutase A and in the virulence of Mycobacterium tuberculosis. Mol Microbiol 48: 453-464.

Briken V. 2012. "With a little help from my friends": Efferocytosis as an antimicrobial mechanism. Cell Host Microbe 12: 261-263.

Briken V. 2013. Mycobacterium tuberculosis genes involved in regulation of host cell death. Adv Exp Med Biol 783: $93-$ 102.

Broz P, Monack DM. 2013. Noncanonical inflammasomes: Caspase-11 activation and effector mechanisms. PLoS Pathog 9: e1003144.

Cadieux N, Parra M, Cohen H, Maric D, Morris SL, Brennan MJ. 2011. Induction of cell death after localization to the host cell mitochondria by the Mycobacterium tuberculosis PE_PGRS33 protein. Microbiology 157: 793-804.

Chen M, Gan H, Remold HG. 2006. A mechanism of virulence: Virulent Mycobacterium tuberculosis strain H37Rv but not attenuated H37Ra, causes significant mitochondrial inner membrane disruption in macrophages leading to necrosis. J Immunol 176: 3707-3716.

Chen M, Divangahi M, Gan H, Shin DS, Hong S, Lee DM, Serhan CN, Behar SM, Remold HG. 2008. Lipid mediators in innate immunity against tuberculosis: Opposing roles of PGE2 and LXA4 in the induction of macrophage death. J Exp Med 205: 2791-2801.
Choi HH, Shin DM, Kang G, Kim KH, Park JB, Hur GM, Lee HM, Lim YJ, Park JK, Jo EK, et al. 2010. Endoplasmic reticulum stress response is involved in Mycobacterium tuberculosis protein ESAT-6-mediated apoptosis. FEBS Lett 584: 2445-2454.

Danelishvili L, Yamazaki Y, Selker J, Bermudez LE. 2010. Secreted Mycobacterium tuberculosis Rv3654c and $\mathrm{Rv} 3655 \mathrm{c}$ proteins participate in the suppression of macrophage apoptosis. PLoS ONE 5: e10474.

Danelishvili L, Everman JL, McNamara MJ, Bermudez LE. 2011. Inhibition of the plasma-membrane-associated serine protease cathepsin $\mathrm{G}$ by Mycobacterium tuberculosis Rv3364c suppresses caspase- 1 and pyroptosis in macrophages. Front Microbiol 2: 281.

Davis JM, Ramakrishnan L. 2009. The role of the granuloma in expansion and dissemination of early tuberculous infection. Cell 136: 37-49.

de Jonge MI, Pehau-Arnaudet G, Fretz MM, Romain F, Bottai D, Brodin P, Honore N, Marchal G, Jiskoot W, England P, et al. 2007. ESAT-6 from Mycobacterium tuberculosis dissociates from its putative chaperone CFP- 10 under acidic conditions and exhibits membrane-lysing activity. J Bacteriol 189: 6028-6034.

Derrick SC, Morris SL. 2007. The ESAT6 protein of Mycobacterium tuberculosis induces apoptosis of macrophages by activating caspase expression. Cell Microbiol 9: 15471555.

DiGiuseppe Champion PA, Cox JS. 2007. Protein secretion systems in mycobacteria. Cell Microbiol 9: 1376-1384.

Divangahi M, Chen M, Gan H, Desjardins D, Hickman TT, Lee DM, Fortune S, Behar SM, Remold HG. 2009. Mycobacterium tuberculosis evades macrophage defenses by inhibiting plasma membrane repair. Nat Immunol 10: 899-906.

Divangahi M, Desjardins D, Nunes-Alves C, Remold HG, Behar SM. 2010. Eicosanoid pathways regulate adaptive immunity to Mycobacterium tuberculosis. Nat Immunol 11: 751-758.

Dorhoi A, Nouailles G, Jorg S, Hagens K, Heinemann E, Pradl L, Oberbeck-Muller D, Duque-Correa MA, Reece ST, Ruland J, et al. 2012. Activation of the NLRP3 inflammasome by Mycobacterium tuberculosis is uncoupled from susceptibility to active tuberculosis. Eur J Immunol 42: 374-384.

Duan L, Gan H, Golan DE, Remold HG. 2002. Critical role of mitochondrial damage in determining outcome of macrophage infection with Mycobacterium tuberculosis. J Immunol 169: 5181-5187.

Edwards KM, Cynamon MH, Voladri RKR, Hager CC, Destefano MS, Tham KT, Lakey DL, Bochan MR, Kernodle DS. 2001. Iron-cofactored superoxide dismutase inhibits host responses to Mycobacterium tuberculosis. Am J Respir Crit Care Med 164: 2213-2219.

Farinacci M, Weber S, Kaufmann SH. 2012. The recombinant tuberculosis vaccine rBCG $\Delta$ ureC::hly ${ }^{+}$induces apoptotic vesicles for improved priming of $\mathrm{CD}^{+}$and $\mathrm{CD}^{+} \mathrm{T}$ cells. Vaccine 30: 7608-7614.

Fratazzi C, Arbeit RD, Carini C, Remold HG. 1997. Programmed cell death of Mycobacterium avium serovar 4infected human macrophages prevents the mycobacteria from spreading and induces mycobacterial growth inhi- 
bition by freshly added, uninfected macrophages. J Immunol 158: 4320-4327.

Galluzzi L, Kepp O, Kroemer G. 2012a. Mitochondria: Master regulators of danger signalling. Nat Rev Mol Cell Biol 13: $780-788$.

Galluzzi L, Vitale I, Abrams JM, Alnemri ES, Baehrecke EH, Blagosklonny MV, Dawson TM, Dawson VL, El-Deiry WS, Fulda S, et al. 2012b. Molecular definitions of cell death subroutines: Recommendations of the Nomenclature Committee on Cell Death 2012. Cell Death Differ 19: 107-120.

Gan H, Lee J, Ren F, Chen M, Kornfeld H, Remold HG. 2008. Mycobacterium tuberculosis blocks crosslinking of annexin-1 and apoptotic envelope formation on infected macrophages to maintain virulence. Nat Immunol 9: 1189-1197.

Gey van Pittius NC, Sampson SL, Lee H, Kim Y, van Helden PD, Warren RM. 2006. Evolution and expansion of the Mycobacterium tuberculosis PE and PPE multigene families and their association with the duplication of the ESAT-6 (esx) gene cluster regions. BMC Evol Biol 6: 95.

Grode L, Seiler P, Baumann S, Hess J, Brinkmann V, Eddine AN, Mann P, Goosmann C, Bandermann S, Smith D, et al. 2005. Increased vaccine efficacy against tuberculosis of recombinant Mycobacterium bovis bacille CalmetteGuérin mutants that secrete listeriolysin. J Clin Invest 115: $2472-2479$

Gross O, Poeck H, Bscheider M, Dostert C, Hannesschlager N, Endres S, Hartmann G, Tardivel A, Schweighoffer E Tybulewicz V, et al. 2009. Syk kinase signalling couples to the Nlrp3 inflammasome for anti-fungal host defence. Nature 459: 433-436.

Hershberg R, Lipatov M, Small PM, Sheffer H, Niemann S, Homolka S, Roach JC, Kremer K, Petrov DA, Feldman MW, et al. 2008. High functional diversity in Mycobacterium tuberculosis driven by genetic drift and human demography. PLoS Biol 6: e311.

Hinchey J, Lee S, Jeon BY, Basaraba RJ, Venkataswamy MM, Chen B, Chan J, Braunstein M, Orme IM, Derrick SC, et al. 2007. Enhanced priming of adaptive immunity by a proapoptotic mutant of Mycobacterium tuberculosis. J Clin Invest 117: 2279-2288.

Hinchey J, Jeon BY, Alley H, Chen B, Goldberg M, Derrick S, Morris S, Jacobs WR Jr, Porcelli SA, Lee S. 2011 Lysine auxotrophy combined with deletion of the SecA2 gene results in a safe and highly immunogenic candidate live attenuated vaccine for tuberculosis. PLOS ONE 6: e15857.

Houben EN, Korotkov KV, Bitter W. 2013. Take five-Type VII secretion systems of mycobacteria. Biochim Biophys Acta doi: 10.1016/j.bbamcr.2013.11.003.

Hsu T, Hingley-Wilson SM, Chen B, Chen M, Dai AZ, Morin PM, Marks CB, Padiyar J, Goulding C, Gingery $\mathrm{M}$, et al. 2003. The primary mechanism of attenuation of bacillus Calmette-Guérin is a loss of secreted lytic function required for invasion of lung interstitial tissue. Proc Natl Acad Sci 100: 12420-12425.

Jayakumar D, Jacobs WR Jr, Narayanan S. 2008. Protein kinase E of Mycobacterium tuberculosis has a role in the nitric oxide stress response and apoptosis in a human macrophage model of infection. Cell Microbiol 10: $365-374$.
Interaction of Mtb with Host Cell Death Pathways

Kaufmann SH. 2013. Tuberculosis vaccines: Time to think about the next generation. Semin Immunol 25: 172-181.

Kausalya S, Somogyi R, Orlofsky A, Prystowsky MB. 2001. Requirement of A1-a for bacillus Calmette-Guèrin-mediated protection of macrophages against nitric oxideinduced apoptosis. J Immunol 166: 4721-4727.

Keane J, Balcewicz-Sablinska MK, Remold HG, Chupp GL, Meek BB, Fenton MJ, Kornfeld H. 1997. Infection by Mycobacterium tuberculosis promotes human alveolar macrophage apoptosis. Infect Immun 65: 298-304.

Kepp O, Galluzzi L, Zitvogel L, Kroemer G. 2010. Pyroptosis-A cell death modality of its kind? Eur I Immunol 40: 627-630.

Kim KH, An DR, Song J, Yoon JY, Kim HS, Yoon HJ, Im HN, Kim J, Kim do J, Lee SJ, et al. 2012. Mycobacterium tuberculosis Eis protein initiates suppression of host immune responses by acetylation of DUSP16/MKP-7. Proc Natl Acad Sci USA 109: 7729-7734.

Koo IC, Wang C, Raghavan S, Morisaki JH, Cox JS, Brown EJ. 2008. ESX-1-dependent cytolysis in lysosome secretion and inflammasome activation during mycobacterial infection. Cell Microbiol 10: 1866-1878.

Kumar D, Narayanan S. 2012. pknE, a serine/threonine kinase of Mycobacterium tuberculosis, modulates multiple apoptotic paradigms. Infect Genet Evol 12: 737-747.

Kurenuma T, Kawamura I, Hara H, Uchiyama R, Daim S, Dewamitta SR, Sakai S, Tsuchiya K, Nomura T, Mitsuyama M. 2009. The RD1 locus in the Mycobacterium tuberculosis genome contributes to activation of caspase1 via induction of potassium ion efflux in infected macrophages. Infect Immun 77: 3992-4001.

Lamkanfi M, Dixit VM. 2010. Manipulation of host cell death pathways during microbial infections. Cell Host Microbe 8: 44-54.

Lee J, Remold HG, Ieong MH, Kornfeld H. 2006. Macrophage apoptosis in response to high intracellular burden of Mycobacterium tuberculosis is mediated by a novel caspase-independent pathway. J Immunol 176: 4267-4274.

Lee J, Repasy T, Papavinasasundaram K, Sassetti C, Kornfeld H. 2011. Mycobacterium tuberculosis induces an atypical cell death mode to escape from infected macrophages. PLOS ONE 6: e18367.

Ligon LS, Hayden JD, Braunstein M. 2012. The ins and outs of Mycobacterium tuberculosis protein export. Tuberculosis (Edinb) 92: 121-132.

Loeuillet C, Martinon F, Perez C, Munoz M, Thome M, Meylan PR. 2006. Mycobacterium tuberculosis subverts innate immunity to evade specific effectors. J Immunol 177: 6245-6255.

Martin CJ, Booty MG, Rosebrock TR, Nunes-Alves C, Desjardins DM, Keren I, Fortune SM, Remold HG, Behar SM. 2012. Efferocytosis is an innate antibacterial mechanism. Cell Host Microbe 12: 289-300.

Mayer-Barber KD, Barber DL, Shenderov K, White SD, Wilson MS, Cheever A, Kugler D, Hieny S, Caspar P, Nunez G, et al. 2010. Caspase-1 independent IL-1 $\beta$ production is critical for host resistance to Mycobacterium tuberculosis and does not require TLR signaling in vivo. J Immunol 184: $3326-3330$.

McElvania Tekippe E, Allen IC, Hulseberg PD, Sullivan JT, McCann JR, Sandor M, Braunstein M, Ting JP. 2010. 
L. Srinivasan et al.

Granuloma formation and host defense in chronic $M y$ cobacterium tuberculosis infection requires PYCARD/ ASC but not NLRP3 or caspase-1. PLoS ONE 5: e12320.

Miao EA, Rajan JV, Aderem A. 2011. Caspase-1-induced pyroptotic cell death. Immunol Rev 243: 206-214.

Miller JL, Velmurugan K, Cowan MJ, Briken V. 2010. The type I NADH dehydrogenase of Mycobacterium tuberculosis counters phagosomal NOX2 activity to inhibit TNFalpha-mediated host cell apoptosis. PLoS Pathog 6: e1000864.

Mishra BB, Moura-Alves P, Sonawane A, Hacohen N, Griffiths G, Moita LF, Anes E. 2010. Mycobacterium tuberculosis protein ESAT-6 is a potent activator of the NLRP3/ASC inflammasome. Cell Microbiol 12: $1046-$ 1063.

Molloy A, Laochumroonvorapong P, Kaplan G. 1994. Apoptosis, but not necrosis, of infected monocytes is coupled with killing of intracellular bacillus Calmette-Guèrin. J Exp Med 180: 1499-1509.

Ng TM, Monack DM. 2013. Revisiting caspase-11 function in host defense. Cell Host Microbe 14: 9-14.

Oddo M, Renno T, Attinger A, Bakker T, MacDonald HR, Meylan PR. 1998. Fas ligand-induced apoptosis of infected human macrophages reduces the viability of intracellular Mycobacterium tuberculosis. J Immunol 160: 5448 5454.

Pan H, Yan BS, Rojas M, Shebzukhov YV, Zhou H, Kobzik L, Higgins DE, Daly MJ, Bloom BR, Kramnik I. 2005. Ipr1 gene mediates innate immunity to tuberculosis. Nature 434: 767-772.

Quarato G, D’Aprile A, Gavillet B, Vuagniaux G, Moradpour D, Capitanio N, Piccoli C. 2012. The cyclophilin inhibitor alisporivir prevents hepatitis $\mathrm{C}$ virus-mediated mitochondrial dysfunction. Hepatology 55: 1333-1343.

Ramakrishnan L. 2012. Revisiting the role of the granuloma in tuberculosis. Nat Rev Immunol 12: 352-366.

Reece ST, Kaufmann SH. 2012. Floating between the poles of pathology and protection: Can we pin down the granuloma in tuberculosis? Curr Opin Microbiol 15: 63-70.

Repasy T, Lee J, Marino S, Martinez N, Kirschner DE, Hendricks G, Baker S, Wilson AA, Kotton DN, Kornfeld H. 2013. Intracellular bacillary burden reflects a burst size for Mycobacterium tuberculosis in vivo. PLoS Pathog 9: e1003190.

Roca FJ, Ramakrishnan L. 2013. TNF dually mediates resistance and susceptibility to mycobacteria via mitochondrial reactive oxygen species. Cell 153: 521-534.

Schaible UE, Winau F, Sieling PA, Fischer K, Collins HL, Hagens K, Modlin RL, Brinkmann V, Kaufmann SH 2003. Apoptosis facilitates antigen presentation to T lymphocytes through MHC-I and CD1 in tuberculosis. Nat Med 9: 1039-1046.

Simeone R, Bobard A, Lippmann J, Bitter W, Majlessi L, Brosch R, Enninga J. 2012. Phagosomal rupture by $M y$ cobacterium tuberculosis results in toxicity and host cell death. PLoS Pathog 8: e1002507.

Sly LM, Hingley-Wilson SM, Reiner NE, McMaster WR. 2003. Survival of Mycobacterium tuberculosis in host macrophages involves resistance to apoptosis dependent upon induction of antiapoptotic Bcl-2 family member Mcl-1. J Immunol 170: 430-437.

Smith J, Manoranjan J, Pan M, Bohsali A, Xu J, Liu J, McDonald KL, Szyk A, LaRonde-LeBlanc N, Gao LY. 2008. Evidence for pore formation in host cell membranes by ESX-1-secreted ESAT-6 and its role in Mycobacterium marinum escape from the vacuole. Infect Immun 76: $5478-5487$.

Sohn H, Kim JS, Shin SJ, Kim K, Won CJ, Kim WS, Min KN, Choi HG, Lee JC, Park JK, et al. 2011. Targeting of Mycobacterium tuberculosis heparin-binding hemagglutinin to mitochondria in macrophages. PLoS Pathog 7: e1002435.

Sridharan H, Upton JW. 2014. Programmed necrosis in microbial pathogenesis. Trends Microbiol. DOI: 10.1016/ j.tim.2014.01.005

Sullivan JT, Young EF, McCann JR, Braunstein M. 2012. The Mycobacterium tuberculosis SecA2 system subverts phagosome maturation to promote growth in macrophages. Infect Immun 80: 996-1006.

Sun J, Singh V, Lau A, Stokes RW, Obregon-Henao A, Orme IM, Wong D, Av-Gay Y, Hmama Z. 2013. Mycobacterium tuberculosis nucleoside diphosphate kinase inactivates small GTPases leading to evasion of innate immunity. PLoS Pathog 9: e1003499.

Tobin DM, Ramakrishnan L. 2013. TB: The yin and yang of lipid mediators. Curr Opin Pharmacol 13: 641-645.

Tobin DM, Vary JC Jr, Ray JP, Walsh GS, Dunstan SJ, Bang ND, Hagge DA, Khadge S, King MC, Hawn TR, et al. 2010. The lta $4 \mathrm{~h}$ locus modulates susceptibility to mycobacterial infection in zebrafish and humans. Cell 140: 717-730.

Tobin DM, Roca FJ, Oh SF, McFarland R, Vickery TW, Ray JP, Ko DC, Zou Y, Bang ND, Chau TT, et al. 2012. Host genotype-specific therapies can optimize the inflammatory response to mycobacterial infections. Cell 148: $434-446$.

Vanlangenakker N, Vanden Berghe T, Vandenabeele P. 2012. Many stimuli pull the necrotic trigger, an overview. Cell Death Differ 19: 75-86.

Velmurugan K, Chen B, Miller JL, Azogue S, Gurses S, Hsu T, Glickman M, Jacobs WR Jr, Porcelli SA, Briken V. 2007. Mycobacterium tuberculosis nuoG is a virulence gene that inhibits apoptosis of infected host cells. PLoS Pathogens 3: el10.

Volkman HE, Pozos TC, Zheng J, Davis JM, Rawls JF, Ramakrishnan L. 2010. Tuberculous granuloma induction via interaction of a bacterial secreted protein with host epithelium. Science 327: 466-469.

Walter K, Holscher C, Tschopp J, Ehlers S. 2010. NALP3 is not necessary for early protection against experimental tuberculosis. Immunobiology 215: 804-811.

Welin A, Eklund D, Stendahl O, Lerm M. 2011. Human macrophages infected with a high burden of ESAT-6-expressing M. tuberculosis undergo caspase-1- and cathepsin B-independent necrosis. PLoS ONE 6: e20302.

Winau F, Kaufmann SH, Schaible UE. 2004. Apoptosis paves the detour path for CD8 $\mathrm{T}$ cell activation against intracellular bacteria. Cell Microbiol 6: 599-607.

Winau F, Weber S, Sad S, de Diego J, Hoops SL, Breiden B, Sandhoff K, Brinkmann V, Kaufmann SH, Schaible UE. 
Interaction of Mtb with Host Cell Death Pathways

2006. Apoptotic vesicles crossprime CD8 T cells and protect against tuberculosis. Immunity 24: 105-117.

Wong KW, Jacobs WR Jr. 2011. Critical role for NLRP3 in necrotic death triggered by Mycobacterium tuberculosis. Cell Microbiol 13: 1371-1384.

Wu K, Koo J, Jiang X, Chen R, Cohen SN, Nathan C. 2012. Improved control of tuberculosis and activation of macrophages in mice lacking protein kinase R. PLoS ONE 7: e30512.
Yang CT, Cambier CJ, Davis JM, Hall CJ, Crosier PS, Ramakrishnan L. 2012. Neutrophils exert protection in the early tuberculous granuloma by oxidative killing of mycobacteria phagocytosed from infected macrophages. Cell Host Microbe 12: 301-312.

Yrlid U, Wick MJ. 2000. Salmonella-induced apoptosis of infected macrophages results in presentation of a bacteria-encoded antigen after uptake by bystander dendritic cells. J Exp Med 191: 613-624. 


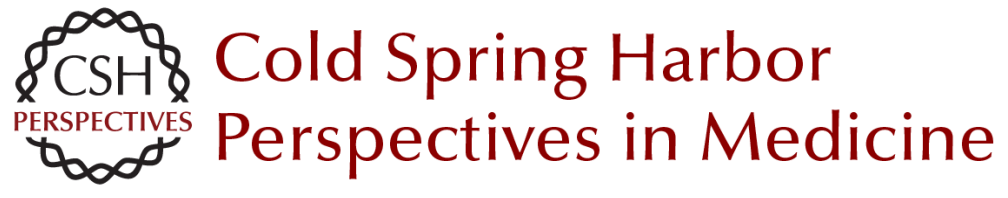

\title{
Interaction of Mycobacterium tuberculosis with Host Cell Death Pathways
}

\author{
Lalitha Srinivasan, Sarah Ahlbrand and Volker Briken
}

Cold Spring Harb Perspect Med 2014; doi: 10.1101/cshperspect.a022459 originally published online June 26, 2014

\section{Subject Collection Tuberculosis}

\section{Transmission and Institutional Infection Control of Tuberculosis Edward A. Nardell \\ Innate and Adaptive Cellular Immune Responses to Mycobacterium tuberculosis Infection Katrin D. Mayer-Barber and Daniel L. Barber}

Tuberculosis Comorbidity with Communicable and Noncommunicable Diseases

Matthew Bates, Ben J. Marais and Alimuddin Zumla

Host-Directed Therapies for Tuberculosis David M. Tobin

Immunity and Immunopathology in the Tuberculous Granuloma

Antonio J. Pagán and Lalita Ramakrishnan

Tuberculosis Drug Development: History and Evolution of the Mechanism-Based Paradigm? Sumit Chakraborty and Kyu Y. Rhee

\section{Genetic Approaches to Facilitate Antibacterial Drug Development Dirk Schnappinger}

The Tuberculosis Drug Discovery and Development Pipeline and Emerging Drug Targets Khisimuzi Mdluli, Takushi Kaneko and Anna Upton
Clinical Aspects of Adult Tuberculosis

Robert Loddenkemper, Marc Lipman and Alimuddin Zumla

Advances in Diagnostic Assays for Tuberculosis Stephen D. Lawn

Diagnosis and Management of Latent

Tuberculosis Infection

Laura Muñoz, Helen R. Stagg and Ibrahim Abubakar

Mycobacterial Growth Iria Uhía, Kerstin J. Williams, Vahid Shahrezaei, et al.

Multidrug-Resistant Tuberculosis and Extensively

Drug-Resistant Tuberculosis

Kwonjune J. Seung, Salmaan Keshavjee and Michael L. Rich

The Mycobacterial Cell Wall--Peptidoglycan and

Arabinogalactan

Luke J. Alderwick, James Harrison, Georgina S. Lloyd, et al.

Tuberculosis and HIV Coinfection Judith Bruchfeld, Margarida Correia-Neves and Gunilla Källenius

Imaging in Tuberculosis Jamshed B. Bomanji, Narainder Gupta, Parveen Gulati, et al.

For additional articles in this collection, see http://perspectivesinmedicine.cshlp.org/cgi/collection/ 